\title{
Affective and pleasurable homeodynamic environments and products: preventive and restorative design for human homeostasis, health and well-being
}

\author{
Keywords \\ Homeodynamic Architectural Environments, Homeodynamic Urban Environments, \\ Homeodynamic Physical and/or Digital Products
}

The concept of "Homeodynamic Environments and Products" is proposed to understand the environment-product-human organism relationship from the inseparable connection between body, mind and spirituality. This concept is coined by the co-founders of the DASMind - UNICAMP [iar. unicamp.br/dasmind]. Three pillars guide the applications of the "Homeodynamic Environments and Products" concept: [1] "Homeodynamic Architectural Environments," preventive and/ or restorative, relate to applying the concept to the study, planning, design and construction of architectural environments. This pillar aims to analyze and understand the user's environments, whether residential, educational, cultural, corporate, commercial, among others, in the body, mind, spirituality relationship. Whether in the design or physical sphere, it reveals the cooperation between the architectural elements and the human organism to restore the body's homeodynamic balance, aiming at its health and well-being. Associated with smart biointerfaces (ZUANON, 2013-2020), this pillar also evaluates and verifies the level of homeodynamic quality of environments in their various purposes: care; cure; labor; exchange of knowledge; leisure; among others. Based on the evaluation, it proposes design solutions conducive to the inner balance of its users, aligned with the purpose of each environment. [2] "Homeodynamic Urban Environments" are supported by urban fabrics, although they do not necessarily represent a simple change of scale, from the architectural scale to the urban scale. In other words, reflecting on the relationships that aim to promo or restore the health and well-being of individuals, as inhabitants or users of the city, in many cases relates to the actual scale of urban design, to the interstice of buildings, to green areas, to small squares and other open urban environments (ZUANON et al., 2020). Moreover, this pillar signals a throwback to classic urban planners who were pioneers in focusing on spatial perception and the relationships of territoriality, privacy, personalization and crowding (MONTEIRO and TURCZYN, 2018). The various humanization studies also provide valuable groundwork for "Homeodynamic Urban Environments," which, alongside smart biointerfaces (ZUANON, 2013-2020), transfer important contributions to design and the implementation of healthy cities. [3] "Homeodynamic Products," whether preventive and/or restorative, relate to the study, design and development of physical, digital and/or physical-digital products capable of "feeling" and "reacting" in real time and empathically to the neuropsychophysiological condition of their users, without interrupting the performance of their routine activities. This pillar operates in deep convergence with smart biointerfaces. In this sense, it correlates contributions from affective computing, cognitive computing, computer vision and wearable computing with the transdisciplinary and complex framework of its concept (ZUANON, 2013-2020). In this intimate connection with the human organism, homeodynamic products provide access to and interaction with the neurobiological scale of affects, emotions and feelings, during different experiences centered on the human being, whether in architectural or urban environments. Furthermore, they enable a consistent interpretation of the body's overall conditions in response to the somatosensory and sensorimotor stimuli produced by those environments. Thus, in establishing a direct relationship with the human organism, "Homeodynamic Environments and Products," whether preventive and/or restorative, in their architectural, urban and object scales, prove to be greatly relevant to life regulation and survival, in both current and future social contexts. 